\title{
Performance Improvement of MIMO MC- CDMA System Using Relay and ITBF
}

\author{
S. Lenin ${ }^{1}$ and N. Tamilarasan ${ }^{2}$ \\ 'Sathyabama University, Old Mahabalipuram Rd, Kamaraj Nagar, \\ Jeppiaar Nagar, Chennai - 600119, Tamil Nadu, India; \\ lenin.ram@gmail.com \\ ${ }^{2}$ Sri Indu Institute of Engineering and Technology, Sheriguda, R.R. Dist, Ibrahimpatnam - 501510, Telengana, India; \\ neithalarasu@gmail.com
}

\begin{abstract}
Objective: In the wireless communication the Inter Symbol Interference (ISI) is a major barrier caused by multipath fading which has a strong negative impact on Bit Error Rate (BER) and affects the high speed data transmission over the wireless channel. Methods: The equalization and channel estimation techniques are available at the receiver (Rx) to reduce the ISI when the channel is affected by multipath propagation effect. Further to decrease the influence of ISI or to improve the quality of the channel condition from the transmitter (Tx), it is reliable to use Relay with Beam-Form (BF) technique because ISI is related to a channel's spatial property. It is natural to expect BF techniques which are often deployed in Multiple Input Multiple Output (MIMO) systems that help to enhance the performance of the system. Findings: In this model, the half duplex Relaying is provided with MIMO antennas with the Amplify and Forward (AF) Relaying protocol. To reduce the ISI and error rate, this study proposes Improved Transmit Beam-Form (ITBF) with equalizationandRelay to be employed with MIMO Multi-Carrier Code Division Multiple Access (MC-CDMA) system. Application: Simulation result depicts the proposed system with ITBF and Relaying is highly reliable to achieve high speed data transmission.
\end{abstract}

Keywords: Equalization and Improved Transmit Beam-Form (ITBF), Inter Symbol Interference (ISI), Multi-Carrier Code Division Multiple Access (MC-CDMA), Multiple Input Multiple Output (MIMO), Relay

\section{Introduction}

The quality of a wireless link is determined by the data rate, transmission range and transmission reliability. These parameters depend on large-scale fading, smallscale fading, interference from other users/system and the user mobility in the network leading to distortion of signal due to excessive multipath delay causing ISI. This results in bit error at the $\mathrm{Rx}$ making realization of high data rate, high capacity and high quality a challenging task $\stackrel{1}{\text {. }}$

This ISI can be overcome by Multiple Input Multiple Output (MIMO) with beam forming and multicarrier modulation. The MIMO antenna at both the ends of a wireless link results in high data rates through spatial multiplexing and increases the spectral efficiency of the system in rich fading and multipath propagation environment by providing spatial diversity. In addition, the MIMO antenna system increases the capacity linearly with the number of transmits-receive antenna pairs without increasing bandwidth and transmitted power. Similarly Orthogonal Frequency Division Multiplexing (OFDM) enables the maximum use of the available bandwidth by designing sub carrier orthogonal to each other. Moreover both of these two techniques are not satisfactory for fast fading environment. To improve the wireless communication quality, the spread spectrum, OFDM and MIMO are combined together which would work on the fast fading environments with reasonable computation complexity.

Further to reduce the influence of ISI and to enhance the system performance in terms of signal to noise ratio,

*Author for correspondence 
the $\mathrm{Tx}$ is provided with $\mathrm{BF}$ in which the antenna patterns or beam can be made towards the desired direction or to suppress the signals in other directions attracting to reduce the effect of interference. Transmit BF is interesting because of its simple design in exploiting the benefits of multiple transmit antennas. The essential requirements for the transmit $\mathrm{BF}$ is the multiple antennas at the $\mathrm{Tx}$, and the use of the measured channel condition between the Tx and the Rx.

Different BF schemes are discussed in literature ${ }^{2-4}$. The distributed BF scheme is discussed ${ }^{5}$ in which single antenna is used in $\mathrm{Tx}$, the $\mathrm{Rx}$ and the Relay nodes. As a result, these schemes do not benefit from the spatial processing at the nodes. Optimal BF design with the Total Power Constraint (TPC) has been studied for MIMO and OFDM however TPC may not be practically appropriate from the high-power amplifier design perspective since the powers allocated to different antennas may vary considerably over time ${ }^{6}$. $\operatorname{In}^{7,8}$ the author discussed the transmit BF for MIMO-OFDM with MRC Rx and partial channel feedback information. In this instantaneous since feedback is considered it does not track the rapid fluctuations of the signal. ICI/ISI aware BF and Noniterative symbol wise $\mathrm{BF}$ for reducing the influence of ISI in MIMO-OFDM system is discussed in ${ }^{9,10}$. Many feedback schemes that dynamically adopt to the distribution of channel are discussed but these methods are complicated to implement in practice.

Moreover to suppress the fading in wireless networks the Relaying techniques are used to overcome the coverage limitations, cost of increasing the number of base stations and more power requirements at the mobile stations for communicating in longer distances at high speed $\frac{11-13}{}$. Many different Relay transmission strategies have been developed ${ }^{14-16}$. Relays can be either full-duplex or half-duplex; full-duplex Relays can transmit and receive at the same time interval while half-duplex Relays cannot. Because full-duplex relays are difficult to implement, practical systems focus on half-duplex Relays $s^{17,18}$. Relays are also classified by how they process the received signal; the most popular Relaying techniques are Decodeand- Forward (DF) and Amplify- and -Forward (AF). DF Relays have higher computational complexity due to the requirement of decoding the signals and are helpful only if they can decode successfully the signals. Compared to DF Relays, AF Relays have the advantage of simple signal processing and end-to-end transparency of data transmission, so it can be used flexibly in heterogeneous networks that contain many nodes with different standard or complexity. The system model and the proposed ITBF is discussed in section 2 and 3 respectively. The simulation result for various MIMO antenna configuration and subcarrier (SC) is discussed in Section 4 and Section 5 which summarizes the conclusion of the work.

\section{System Model}

MIMO MC-CDMA system with Relay and ITBF is shown in Figure 1 where the $\mathrm{Rx}$ and Relay node receive the signal through Tx. The Tx, Rx and Relay nodes are provided with $\mathrm{N}_{s}, \mathrm{~N}_{\mathrm{d}}$ and $\mathrm{N}_{\mathrm{r}}$ antennas respectively. To avoid the interference between direct path and Relay links, the communication process requires two time slots. Once the signal is modulated by $N_{C}$ SCs, $N_{b}^{(n)}, n=1, \ldots . ., N_{c}$ as the number of symbols in the $\mathrm{n}^{\text {th }}$ SCs is transmitted to the Relay as well as to the $\mathrm{Rx}$ in the 1st time slot. The Relay node retransmits the amplified version of the signal to the $\mathrm{Rx}$ in the 2nd time slot.

Figure 2 shows the Tx structure of MIMO MC CDMA system comprising of channel equalization, channel estimation, ITBF and Relay In the Tx the data stream is multiplied by a spreading sequence after modulation and the pilot signals are added for estimating channel status in the Rx. At the end,the signals are beam formed and directed to both the Relay and $\mathrm{Rx}$ through multiple antennas. In Figure 3, the received signal is demodulated using Fast Fourier Transform (FFT) and the pilot symbols are used for obtaining transfer function of the channel which in turn is used for equalization and beamforming. With the knowledge of estimated channel, data symbols are recovered at the $\mathrm{Rx}$.

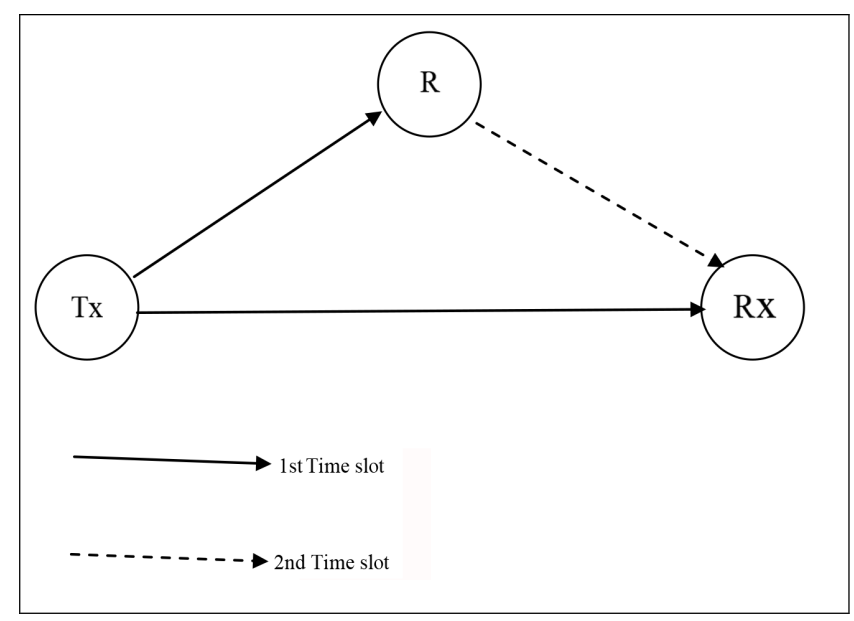

Figure 1. Three node AF relay. 


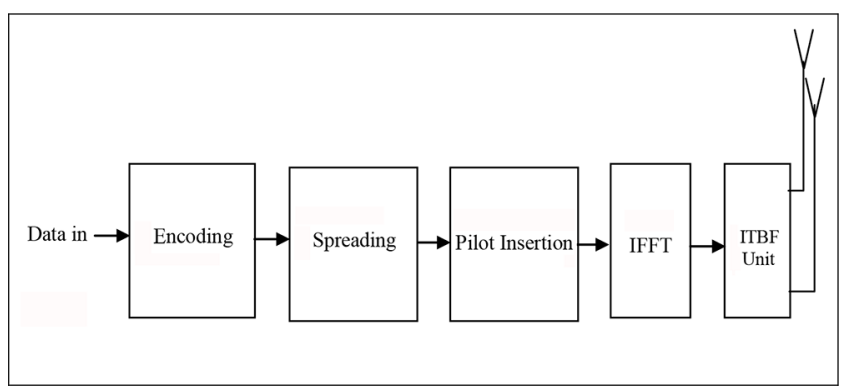

Figure 2. Tx structure of MIMO MC-CDMA.

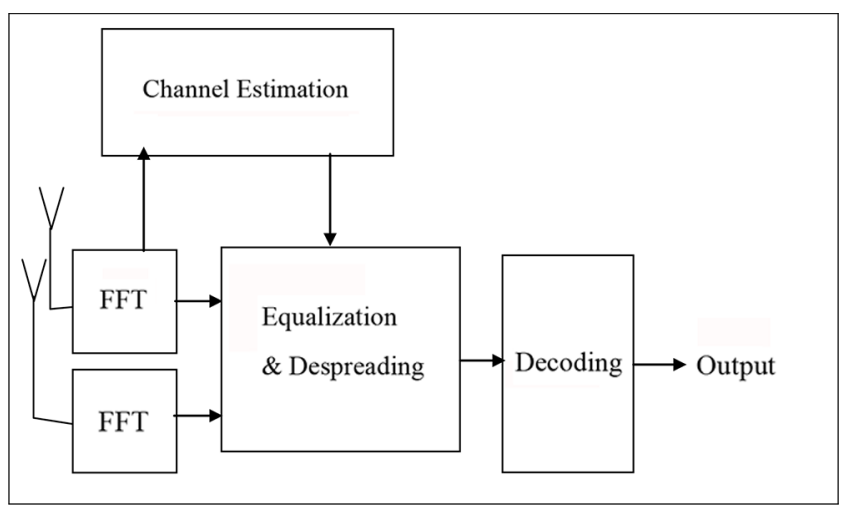

Figure 3. Rx structure of MIMO MC CDMA.

\section{ITBF and Relay}

Even though Channel estimation based on equalization is employed in the $\mathrm{Rx}$ to tackle the effect of multipath and reduce ISI, it is better to BF the signals from the Tx antennas based on Channel State Information (CSI) to further improve the quality of the received signal. In this work, ITBF technique is proposed, which utilize the CSI details obtained from the modified channel estimation algorithms. In the proposed ITBF technique, BF is dynamically carried out i.e. the rate at which the BF vectors are updated matches with the rate at which channel varies. Hence it is ensured, the moment there is a change in CSI, and change in BF vector is invoked. The logic used in ITBF is depicted in the flowchart is shown in Figure 4.

This model consists of a Relay with two antenna, source node with $\mathrm{N}_{\mathrm{T}}$ transmitting antenna and destination with $\mathrm{N}_{R}$ receiving antenna. The transmission is organized in two time slots. The Tx transmits a data packet which is received by the Rx and Relay in the 1st time slot. Similarly the Relay amplifies and retransmits the signal to the $\mathrm{Rx}$ in the 2nd time slot. At the $\mathrm{Rx}$, the packets received during the 1st and 2 nd time slots are combined, processed and detected.

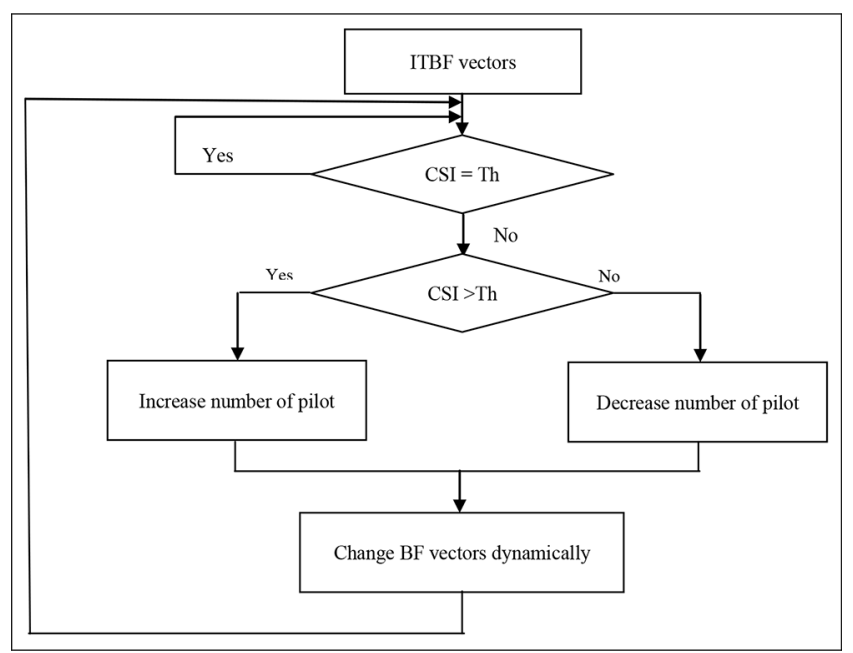

Figure 4. Flowchart of ITBF.

The discrete time Channel Impulse Responses (CIR) between the Tx and Relay node $g_{i}[k], 0 \leq k \leq L_{g}-1$, between Relay node and the $\mathrm{Rx}, \mathrm{hi}[k], 0 \leq k \leq L_{h}-1$, and between the $\operatorname{Tx}$ and $\operatorname{Rxf}_{\mathrm{i}}[k], 0 \leq k \leq L_{f}-1$, contains the combined effect of transmit pulse shaping, the continuous time channel, receive filtering and sampling. Here $L_{g}$, $L_{h}$ and $L_{f}$ denotes the length of the Tx-Relay, the Relay-Rx, and the Tx-Rx channels respectively.

In the first interval a signal $x \in \mathrm{C}$ with power $P=$ $E\left\{[x]^{2}\right\}$ is sent from the Txthrough flat fading channel $g_{i}$ the channel element in $g_{i}$ are independent Rayleigh fading distributed with variance $\sigma^{2}$. The signal received by the Relay nodewith Additive white Gaussian noise $n[k]$ can be expressed as:

$$
y_{i}[k]=\sum_{i=1}^{N_{T}} g_{i}[\mathrm{k}]^{\star} x[k]+n[k]
$$

During the 2nd time interval the signal transmitted by the $\mathrm{i}^{\text {th }}$ Relay antenna can be expressed as:

$$
\begin{aligned}
t_{i}(k) & =\sum_{i=1}^{2} W_{i}[k]^{\star} y_{i}[k] ; \\
& =\sum_{i=1}^{2} W_{i}[k]^{\star} g_{i}[k]^{\star} x[k]+W_{i}[k]^{\star} n[k]
\end{aligned}
$$

Where $W_{i}[k]$ beam form matrix of MIMO AF Relay

\subsection{Equalization at Destination Node}

During the 1st time slot the signal received by the $\mathrm{Rx}$ is given by:

$$
d_{1}(k)=\sum_{i=1}^{N_{T}} f_{i}[k] * x[k]+n_{0}[k]
$$


During the 2nd transmission time slot the signal received at the $\mathrm{Rx}$ is given by:

$$
\begin{gathered}
d_{2}(k)=\sum_{i=1}^{2} \mathrm{~h}_{\mathrm{i}}[k]^{\star} t_{i}(k)+n_{1}[k] \\
=\sum_{i=1}^{2} \mathrm{~h}_{\mathrm{i}}[k]^{\star} W_{i}[k]^{*} g_{i}[k]^{\star} x[k] \\
+\sum_{i=1}^{2} \mathrm{~h}_{\mathrm{i}}[k]^{\star} W_{i}[k]^{\star} \mathrm{n}[k]^{\star} n_{1}[k] \\
=h_{e q}[k]^{\star} x[k]^{\star} \mathrm{n}[k]^{\star} n_{1}[k] \\
h_{e q}=\sum_{i=1}^{2} \mathrm{~h}_{\mathrm{i}}\left[k{ }^{\star} W[k]_{i}^{*} g_{i}[k]\right.
\end{gathered}
$$

Where $n_{0}[k]$ and $n_{1}[k]$ is AWGN noise with variance? $\sigma_{n}^{2}$

\subsection{Feedback Channel}

We assume that the Rx estimates the Relay-Rx CIRs $\mathrm{h}_{\mathrm{i}}[k]$, $0 \leq k \leq L_{h}, 1<i \leq 2$ and the Tx-Rx $\operatorname{CIR~}_{\mathrm{i}}[k], 0 \leq k \leq L_{f}-1$ during training phase. The $\mathrm{Rx}$ directly estimates the combined CIR of the source-Relay and Relay-Rx channels $\mathrm{h}_{\mathrm{i}}[k]^{*} g_{i}[k]$ if the Relay node retransmits the training signal received from the Tx. The Rx can extract $g_{i}[k]$ from $\mathrm{h}_{\mathrm{i}}[k]^{*} g_{i}[k]$ and $\mathrm{h}_{\mathrm{i}}[k]$ via de-convolution.

\section{Result and Discussion}

The proposed system of MIMO MC-CDMA with Relay and ITBF are simulated in MATLAB with the parameters given in Table 1 and BER is calculated by varying energy per bits to spectral noise density $\left(\mathrm{E}_{\mathrm{b}} / \mathrm{N}_{\mathrm{o}}\right)$. Figure 5 shows the BER performance of the MIMO-MC CDMA using 64 SCs with $2 \times 1 \times 2$ and $2 \times 2 \times 2$ MIMO antennas configuration. From the result it is clear that the BER is reduced by improving gain in the desired direction and rejects interference using relay with ITBF. Further the simula-

Table 1. Simulation parameters

\begin{tabular}{|l|l|}
\hline \multicolumn{1}{|c|}{ Spreading Codes } & \multicolumn{1}{c|}{ Walsh Hadamard code } \\
\hline Number of SCs & $64 / 128$ \\
\hline Channel & Rayleigh fading \\
\hline Modulation & $16 \mathrm{QAM}$ \\
\hline Antennas configuration & 2x2/4x4 \\
\hline Equalization/Estimation & MMSE/Pilot \\
\hline
\end{tabular}

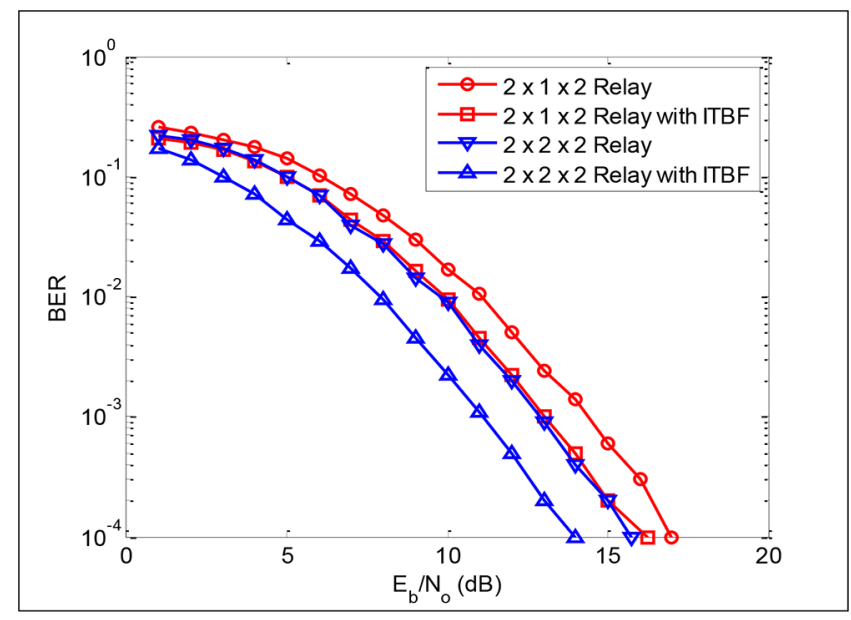

Figure 5. Simulation result of MIMO MC CDMA with relay and ITBF $(2 \times 2,64$ SCs).

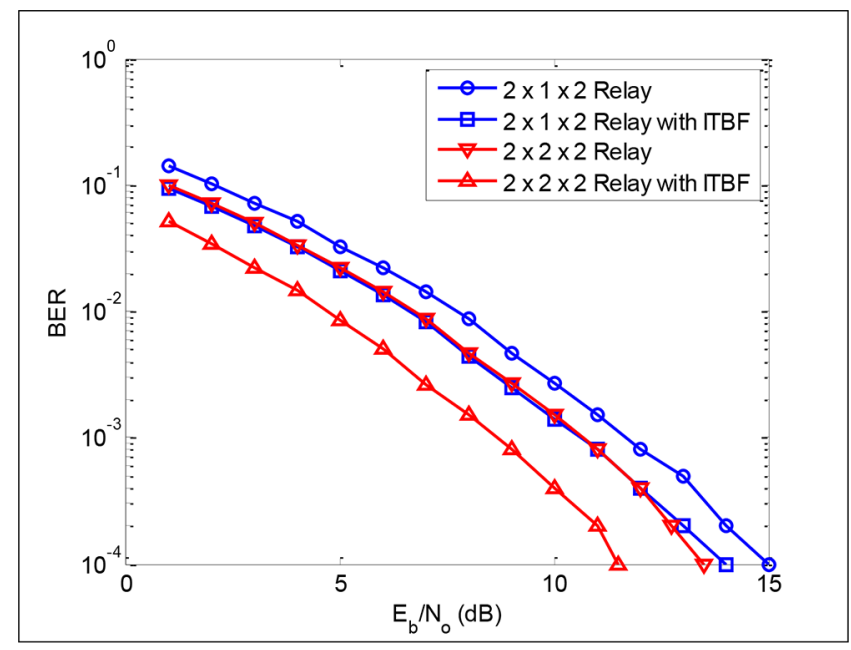

Figure 6. Simulation result of MIMO MC CDMA with relay and $\operatorname{ITBF}(2 \times 2,128$ SCs $)$.

tion is repeated by increasing the SCs(128SCs) and the results aredisplayed in Figure 6 in which the performance is improved due to frequency diversity and space diversity offered by the relays.

The performance of $4 \times 4$ antennas configuration for 64 SCs and 128 SCs are shown in Figure 7 and 8 respectively and it is observed that the performance of the system is increased due to antenna diversity and frequency diversity. More detailed reports are shown in Table 2, where $\mathrm{Eb} / \mathrm{No}$ requirement for a target BER of $10^{-3}$ is given for various antenna configurations, SC, Relays and ITBF. From Table 2 it is quite clear, as Relay antenna 


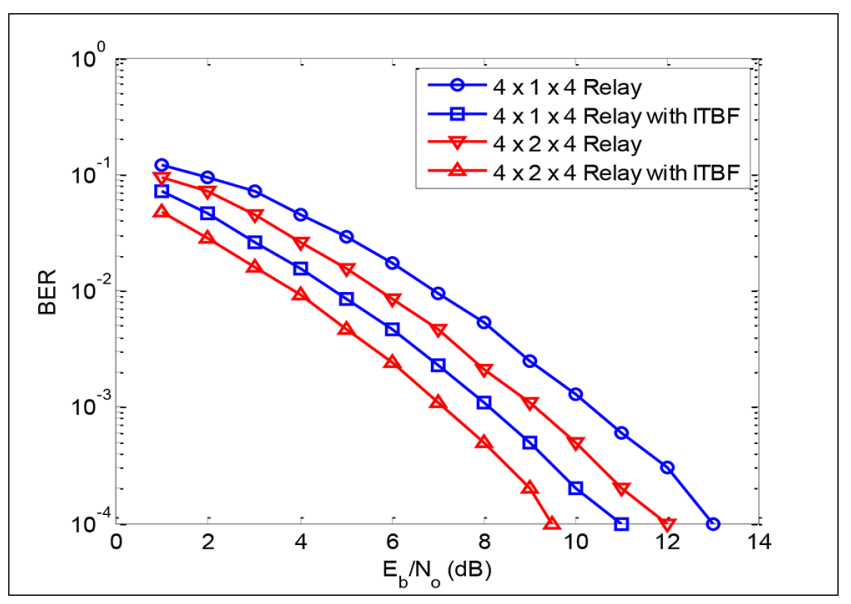

Figure 7. Simulation result of MIMO MC CDMA with relay and ITBF $(4 \times 4,64$ SCs $)$.

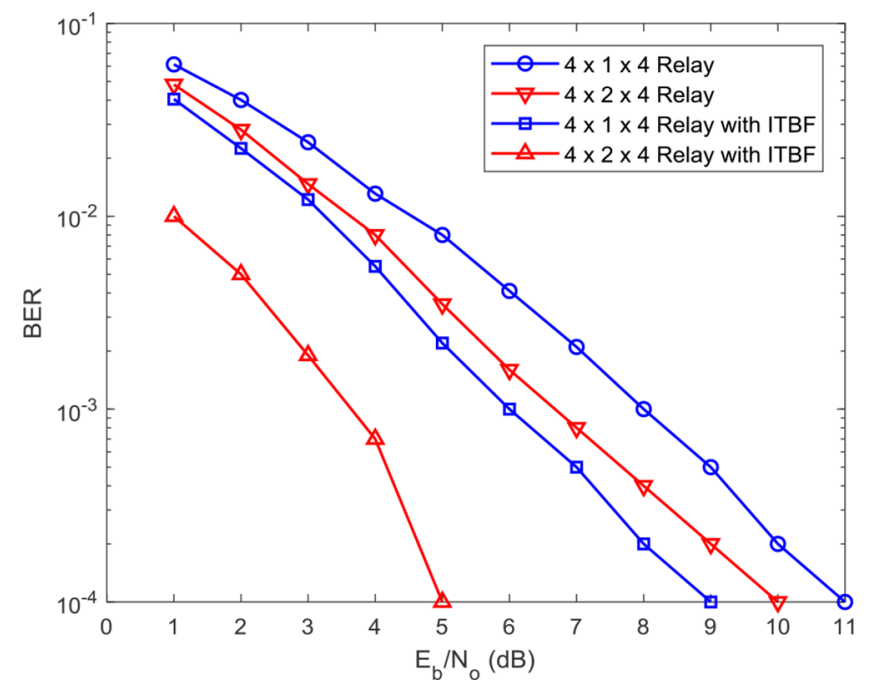

Figure 8. Simulation result of MIMO MC CDMA with relay and ITBF $(4 \times 4,128 \mathrm{SCs})$.

is increased from 1 to 2 , approximately 1 to $2.5 \mathrm{~dB}$ less $\mathrm{E}_{\mathrm{b}} / \mathrm{N}_{\mathrm{o}}$ is required to maintain a target BER of $10^{-3}$. This due to the space diversity offered by Relays. Also, it can be noted as SC increases from 64 to 128 approximately 2 to $3 \mathrm{~dB}$ less $\mathrm{E}_{\mathrm{b}} / \mathrm{N}_{\mathrm{o}}$ is required for the target $\mathrm{BER}$ of $10^{-3}$ and this is due to the frequency diversity offered by SCs.

For example in Table 2 Relay with two antennas $\mathrm{E}_{\mathrm{b}} / \mathrm{N}_{\mathrm{o}}$ decreases by $1.3 \mathrm{~dB}$ then the Relay with single antenna at the target BER of $10^{-3}$ due to space diversity. Similarly Relay (2 antenna) with BF reduces $\mathrm{E}_{\mathrm{b}} / \mathrm{N}_{\mathrm{o}}$ by $1.3 \mathrm{~dB}$ compared to Relay with single antenna. From Table 2 it is also observed that performance can also progress by increasing the frequency diversity and antenna diversity. With antenna configuration of $2 \times 2$, the increasing SC from 64 to 128 the $\mathrm{E}_{\mathrm{b}} / \mathrm{N}_{\mathrm{o}}$ requirement is reduced by $2.5 \mathrm{~dB}$ increasing the SC from 64 to 128 . Similarly for 64 SC the increasing antenna configuration from $2 \times 2$ to $4 \times 4$ the $\mathrm{E}_{\mathrm{b}} / \mathrm{N}_{\mathrm{o}}$ requirement is reduced by $4.1 \mathrm{~dB}$.

\section{Conclusion}

This study discusses about ITBF and Relays in the MIMO MC-CDMA with different SCs to improve the quality of wireless link. The performance of the system from Tx side is improved by ITBF using narrow beam directed towards the desired direction, and channel quality of the wireless link is increased by AF Relay node between the Tx and the Rx. The result shows after incorporating Relay in MIMO MC-CDMA system, the $\mathrm{E}_{\mathrm{b}} / \mathrm{N}_{\mathrm{o}}$ requirement decreases by $47 \%$ (approximately) with increasing antenna configuration from $2 \times 2$ to $4 \times 4$ for $128 \mathrm{SC}$. Further $\mathrm{E}_{\mathrm{b}} / \mathrm{N}_{\mathrm{o}}$ is decreased using BF by suppressing the interference from other channel. It can also be inferred that the increase in number of antennas in the Tx and RX, increases in the number of SCs and the Relay improves the BER performance due to the diversity and multiplexing gain.

Table 2. Performance of MIMO MC CDMA system at the target BER of 10-3 byantenna configuration

\begin{tabular}{|c|c|c|c|c|}
\hline \multirow[t]{2}{*}{ Antenna configuration/SCs } & \multicolumn{2}{|c|}{ Relay } & \multicolumn{2}{|c|}{ Relay with ITBF } \\
\hline & 1 AntennaE $\mathrm{b}_{\mathrm{b}} / \mathrm{N}_{\mathrm{o}}(\mathrm{dB})$ & 2 Antenna $\mathrm{E}_{\mathrm{b}} / \mathrm{N}_{\mathrm{o}}(\mathrm{dB})$ & 1 Antenna $\mathrm{E}_{\mathrm{b}} / \mathrm{N}_{\mathrm{o}}(\mathrm{dB})$ & 2 Antenna $\mathrm{E}_{\mathrm{b}} / \mathrm{N}_{\mathrm{o}}(\mathrm{dB})$ \\
\hline $2 \times 2 / 64$ SCs & 14.3 & $13(1.3 \downarrow)$ & $13(1.3 \downarrow)$ & $11.1(1.9 \downarrow)$ \\
\hline $2 \times 2 / 128$ SCs & 11.8 & $10.5(1.3 \downarrow)$ & $10.6(1.2 \downarrow)$ & $8.6(1.9 \downarrow)$ \\
\hline $4 \mathrm{x} 4 / 64$ SCs & 10.2 & $9.2(1 \downarrow)$ & $8(2.2 \downarrow)$ & $7.2(2 \downarrow)$ \\
\hline $4 \mathrm{x} 4 / 128 \mathrm{SCs}$ & 8 & $6.2(1.8 \downarrow)$ & $6(2 \downarrow)$ & $3.8(2.4 \downarrow)$ \\
\hline
\end{tabular}




\section{Reference}

1. Rusek F, Prlja A. Optimal channel shortening for MIMO and ISI channels, IEEE Transactions on Wireless Communications. 2012; 11(2):810-18. https://doi.org/10. 1109/TWC.2011.121911.110809.

2. Ju H, Lim S, Kim D, Vincent Poor H, Hong D. Full duplexity in beamforming-based multi-hop relay networks, IEEE Journal on Selected Areas on Communication. 2012; 30(8):1554-65. https://doi.org/10.1109/JSAC.2012.120922.

3. Wu P, Schober R. Cooperative beamforming for singlecarrier frequency-domain equalization systems with multiple Rs, IEEE Transactions on Communications. 2012; 11(6): 2276-86.

4. Chalise BK, Vandendorpe L, Zhang YD, Amin MG. Local CSI based selection beamforming for amplify-and-forward MIMO relay networks, IEEE Transactions on Signal Processing. 2012; 60(5):2433-46. https://doi.org/10.1109/ TSP.2012.2184535.

5. Havary-Nassab V, Shahbazpanahi S, Grami A, Luo ZQ. Distributed beamforming for Relay networks based on second-order statistics of the channel state information, IEEE Transactions on Signal Processing. 2008; 56(9): 4306-16. https://doi.org/10.1109/TSP.2008.925945.

6. Zheng X, Xie Y, Li J, Stoica P. MIMO transmits beamforming under uniform elemental power constraint, IEEE Transactions on Signal Processing. 2007; 55(11):5395-406. https://doi.org/10.1109/TSP.2007.896058.

7. Lopez-Martinez FJ, Martos-Naya E, Paris JF, Goldsmith AJ. BER Analysis for MIMO-OFDM Beamforming with MRC Under Channel Prediction and Interpolation Errors. Proceedings of IEEE GLOBECOM; 2009. p. 1-7. https:// doi.org/10.1109/GLOCOM.2009.5426059.

8. Zhou S, Giannakis GB. Optimal Txeige Beamforming and Space-Time Block Coding Based on Channel Mean Feedback, IEEE Transactions on Signal Processing. 2002; 50(10):2599-613. https://doi.org/10.1109/TSP.2002.803355.

9. Sun X, Wang Q, Cimini LJ, Greenstein LJ, Chan DS. ICI/ISIAware beamforming for MIMO-OFDM wireless systems, IEEE Transactions on Wireless Communications. 2012; 11(1):378-85. https://doi.org/10.1109/TWC.2011.112311. 111705.
10. Hyun-Ho Lee, Young-Chai Ko. Non-Iterative symbol-wise beamformingforMIMO-OFDM systems, IEEETransactions on Wireless Communications. 2012;11(10):3788-98.https:// doi.org/10.1109/TWC.2012.081612.120406.

11. Mehrizi S, Mahboobi B. Cooperative MIMO relay beamforming in overloaded DS/CDMA multicast networks, IET Communications. 2017; 11(3):344-54. https://doi.org/ 10.1049/iet-com.2016.0610.

12. Li C, Chen, Wang Y. Outage analysis of the full-duplex decode-and-forward two-way RSystem, IEEE Transactions on Vehicular Technology. 2017; 66(5):4073-86. https://doi. org/10.1109/TVT.2016.2610004.

13. Lee I, Kim D. Coverage extension and power allocation in dual hop space-time transmission with multiple antennas in each node, IEEE Transactions on Vehicular Technology. 2007; 56(6):3524-32. https://doi.org/10.1109/ TVT.2007.901901.

14. Waqar O, Imran MA, Dianati M, Tafazolti R. Energy consumption analysis and optimization of BER constrained amplify and forward network, IEEE Transactions on Vehicular Technology. 2014; 63(3):1256-69. https://doi. org/10.1109/TVT.2013.2284405.

15. Lee K, Li C, Yiwang T, Li H. Performance analysis of dualhop amplify-and-forward systems with multiple antennas and co-channel interference, IEEE Transactions on Wireless Communications. 2014; 13(6):3070-87. https://doi.org/10. 1109/TWC.2014.042814.130047.

16. Sanguinetti L, D'Amico AD, Rong Y. On the design of amplify-and-forward MIMO-OFDM relay systems with QoS requirements specified as schur-convex functions of the MSEs, IEEE Transactions on Vehicular Technology. 2013; 62:4871-77. https://doi.org/10.1109/TVT.2012.2236370.

17. Wang JS. Cooperative relaying of superposition coding with simple feedback for layered source transmission, IEEE Transactions on Communications. 2013; 61(11):4448-81. https://doi.org/10.1109/TCOMM.2013.100313.120764.

18. Peters SW, Panah AY, Truong KT, Heath W. Relay architectures for 3GPP LTE-Advanced. EURASHIP Communication Networking; 2009. 